\title{
Patterned Poly(dopamine) Films for Enhanced
}

\section{Cell Adhesion}

Xi Chen, ${ }^{\dagger}$ Christina Cortez-Jugo, ${ }^{\dagger}$ Gwan H. Choi, ${ }^{\ddagger}$ Mattias Björnmalm, ${ }^{\dagger}$ Yunlu Dai, ${ }^{\dagger}$ Pil J. Yoo $^{\ddagger, s}$ and Frank Caruso ${ }^{\dagger, *}$

${ }^{\dagger}$ Australian Research Council (ARC) Centre of Excellence in Convergent Bio-Nano Science and Technology, and the Department of Chemical and Biomolecular Engineering, The University of Melbourne, Parkville, Victoria 3010, Australia

${ }^{\ddagger}$ School of Chemical Engineering, Sungkyunkwan University, Suwon 440-746, Republic of Korea

${ }^{\S}$ Sungkyunkwan Advanced Institute of Nanotechnology, Sungkyunkwan University, Suwon 440-746, Republic of Korea

E-mail: fcaruso@unimelb.edu.au. Phone: +61 38344 3461. Fax: +61 383444153.

\begin{abstract}
Engineered materials that promote cell adhesion and cell growth are important in tissue engineering and regenerative medicine. In this work, we produced poly(dopamine) (PDA) films with engineered patterns for improved cell adhesion. The patterned films were synthesized via the polymerization of dopamine at the air/water interface of a floating bed of spherical particles. Subsequent dissolution of the particles yielded free-standing PDA films with tunable geometrical patterns. Our results show that these patterned PDA films significantly enhance the adhesion of both cancer cells and stem cells, thus showing promise as substrates for cell attachment for various biomedical applications.
\end{abstract}




\section{Introduction}

Artificial materials that enable the attachment of cells for cell growth and proliferation play an important role in tissue engineering and regenerative medicine. ${ }^{1-3}$ Such substrates and scaffolds can be engineered to provide a template for the hierarchal growth of cells or as cell supports, facilitating attachment and potentially serving as reservoirs for growth factors that regulate cell growth and function. Cell adhesion onto scaffold biomaterials has been described to occur at the macro- and nanoscale, ${ }^{4}$ and can be modulated by the mechanical properties, chemical composition, surface properties and topography of the substrate. ${ }^{5-9}$ For example, it has been demonstrated that cell adhesion on soft hydrogel films is influenced by the stiffness or elastic modulus of the films. ${ }^{10}$ Surfaces with honeycomb-like structures, ${ }^{11}$ grooves, ridges, wells and pillar-like structures have also been shown to influence cell

adhesion. ${ }^{12-14}$ At the nanoscale, the presence of receptors, such as RGD or other ligands that mimic the extracellular matrix, can control cell attachment, behavior and function. ${ }^{4}$ These provide examples of the complex interaction between cells and substrates that govern cellular adhesion, and subsequently, growth and proliferation. These examples also highlight the importance of considering material properties at the macro- and nanoscale when designing substrate materials.

Poly(dopamine) (PDA), formed by the oxidative polymerization of dopamine (DA) in alkaline conditions, is a melanin-like biopolymer present in living organisms. ${ }^{15}$ Inspired by adhesive proteins in mussels, Lee et al. showed a surface modification method based on DA polymerization onto a wide range of organic and inorganic substrates, including metals, oxides, polymers, semiconductors, and ceramics. ${ }^{16}$ Further, the resulting PDA surface coating remains reactive to amine and thiol groups via Schiff base formation or Michael addition, which provides a facile approach to post-modify surfaces with various functional 
molecules. ${ }^{17}$ The advantage of PDA coatings resides in their simplicity, versatility, and biocompatibility. ${ }^{15}$ Studies have demonstrated that PDA-based materials are promising candidates as drug carriers, ${ }^{18-20}$ polymer scaffolds in tissue engineering, including for bone or muscle regeneration, neural formation, and as vascular stents. ${ }^{15,21-23}$ These results demonstrate the potential of PDA coatings for biomedical applications.

Herein, we use a templated assembly approach to fabricate PDA films ${ }^{24}$ with controlled surface topology and investigate the adhesion of cancer cells and stem cells on these engineered surfaces (Scheme 1). The PDA films produced had an indented topography, with the size of the indentations tunable by the diameter of the spherical template used to produce the films. Cell adhesion studies were performed and a substantial increase in cell adhesion (44\% increase for HeLa cells and 183\% increase for mesenchymal stem cells) was observed on the patterned PDA films compared with non-patterned PDA films. These results demonstrate the potential of this simple and versatile technique for creating patterned PDA surfaces with enhanced cell adhesion, with potential application as cell supports in tissue engineering and biomedical research.

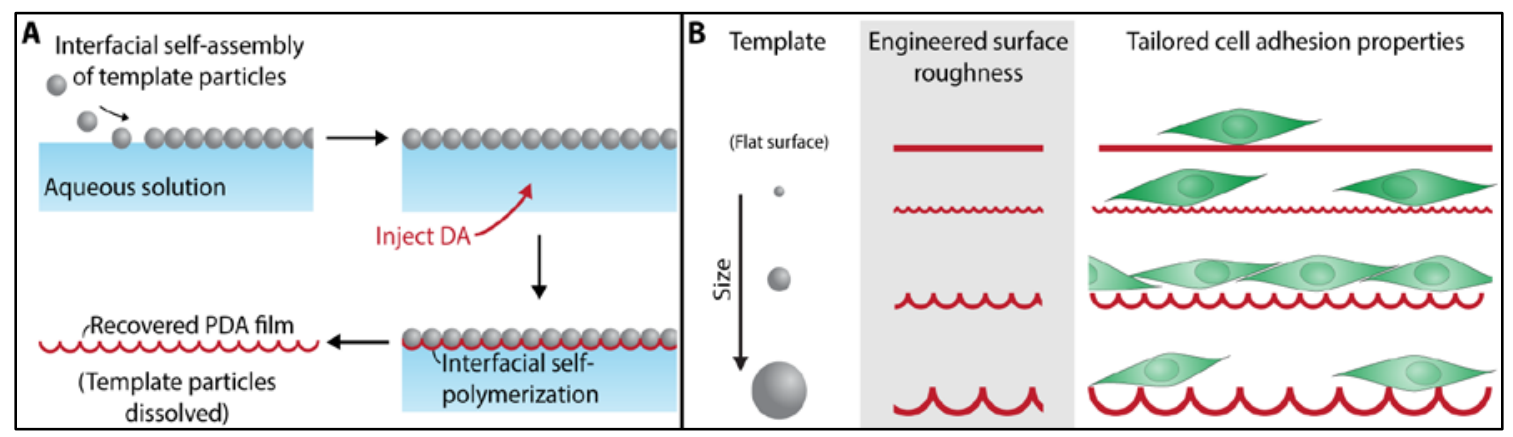

Scheme 1. (A) Preparation of patterned PDA films through interfacial self-polymerization of DA on partially immersed template particles. (B) The surface roughness can be controlled by changing the template particle size, resulting in PDA films with tailored cell adhesion properties. 


\section{Results and Discussion}

Hexagonally close-packed monolayers of patterned PDA films were produced in three steps via particle-templated assembly. Firstly, monodispersed polystyrene (PS) particles, with density of $1.05 \mathrm{~g} \mathrm{~cm}^{-3}$ and a diameter of $0.5 \mu \mathrm{m}$, were added to an aqueous solution and allowed to float to the surface, where they formed a close-packed array (Scheme 1). Secondly, DA monomer solution was injected into the aqueous medium, which triggered selfpolymerization of DA on the surfaces of the particles at the liquid/solid interface. This resulted in an anisotropic modification of the floating or partially immersed PS templates. Finally, the PS particles partially coated with PDA (denoted PDA/PS films) were removed from the aqueous solution and deposited on a substrate. The sacrificial PS templates were then dissolved with the addition of tetrahydrofuran, yielding patterned PDA films.

Scanning electron microscopy (SEM) was employed to investigate the surface morphology of the PDA/PS films and the corresponding PDA films (Figure 1A, B). SEM shows the monolayer arrangement of PS particles within the PDA/PS film, with the particles exhibiting a hexagonally close-packed pattern with minimal defects over $4 \times 5 \mu^{2}$ (Figure 1A). After template removal, the underlying PDA film retained its structure, leaving "grooves" or "indentations" where the PS particles had been (Figure 1B). This is consistent with our

previous study on using spherical templates to obtain hollow PDA polymer capsules. ${ }^{18-20}$ The capsules maintained the size and the shape of the template particles used, without substantial swelling or shrinkage occurring in solution, which indicated that hollow PDA-based materials were mechanically stable. AFM was performed to image the topography of the patterned PDA films (Figure 1C). Three-dimensional surface plots and two-dimensional contour plots show regular patterns or grooves on the PDA film, further demonstrating the fidelity of the templated assembly approach. 

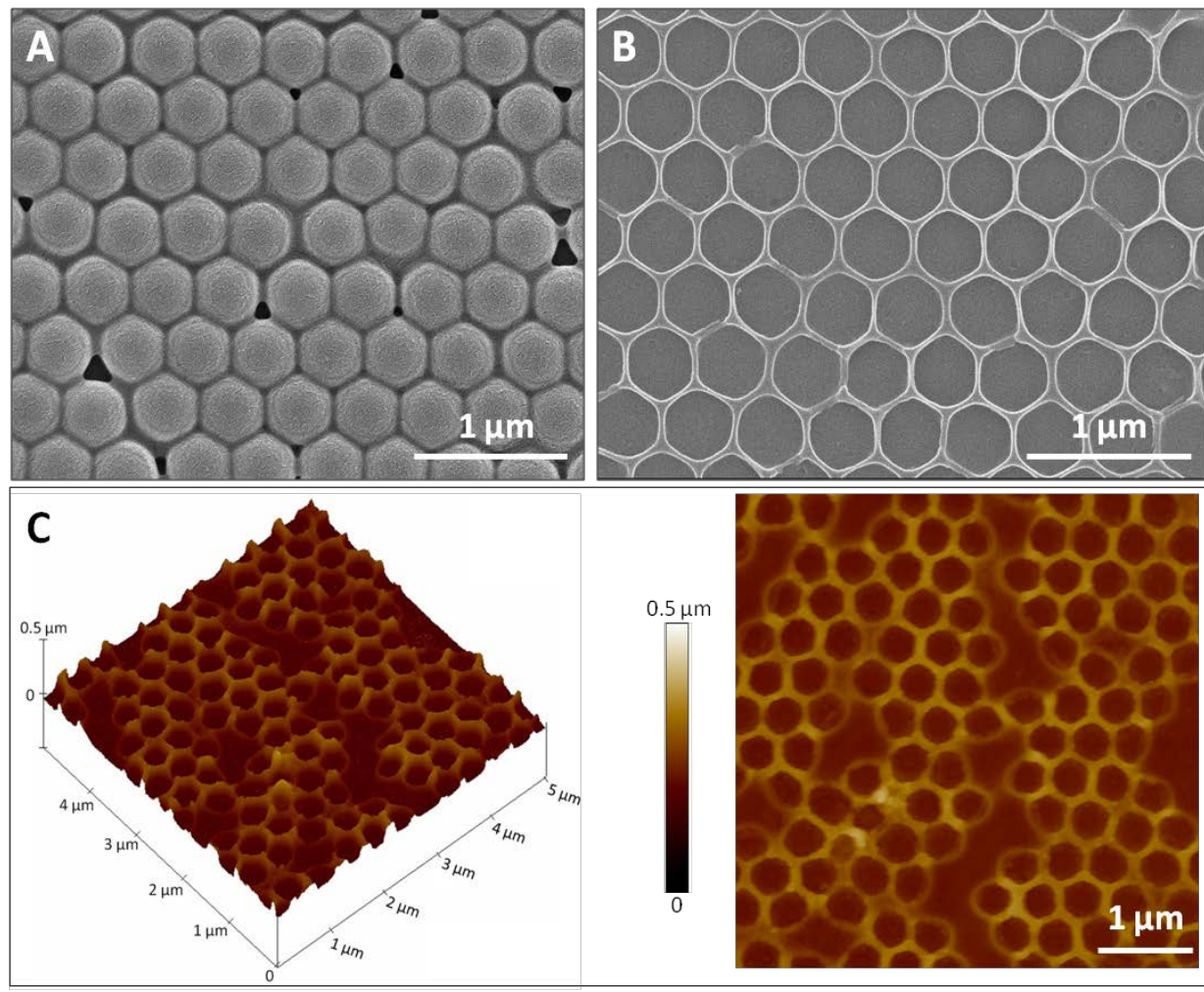

Figure 1. SEM images showing the topography of (A) a PDA/PS film, (B) a patterned PDA film after dissolution of the spherical PS templates, and (C) an AFM image of a patterned PDA film. All the films were derived using PS template particles with diameters of $0.5 \mu \mathrm{m}$.

At the nanoscale, the surface structure or topography of the PDA films could be controlled by using PS templates of different sizes. PS particles of diameters $0.8 \mu \mathrm{m}$ and 1.7 $\mu \mathrm{m}$ were used as templates to produce patterned PDA films with different groove sizes. The corresponding PS/PDA films before template removal are shown in Figure S1. SEM and AFM images show that (as with the $0.5 \mu \mathrm{m}$ particles) the size and shape of the template used are retained (Figure 2). However, it is noted that the larger template particles resulted in less uniform films with increased surface roughness, which may be due to the grooves being deeper and the surrounding hexagonal walls being prone to tear and/or collapse (Figure S2).

A line profile analysis of the AFM data was used to investigate the depth of the indentations (Figure S3). For $0.5 \mu \mathrm{m}$ templates, the depth was found to be around $100 \mathrm{~nm}$, 
which is approximately $20 \%$ of the diameter of the particle used. For $0.8 \mu \mathrm{m}$ and $1.7 \mu \mathrm{m}$ templates, grooves with average depths of around $130 \mathrm{~nm}$ and $220 \mathrm{~nm}$, respectively, were obtained. This corresponds to approximately $16 \%$ and $13 \%$ of the diameters of the $0.8 \mu \mathrm{m}$ and $1.7 \mu \mathrm{m}$ particles, respectively. Nevertheless, taken together these results demonstrate that patterned PDA films with controllable groove size can be obtained through the partial polymerization of DA at the liquid/solid interface of floating sacrificial particle templates. Other methods for fabricating similar honeycomb-like structures include the breath figure technique based on the self-arrangement of water droplets in the presence of polymer and solvent, and facilitated by air flow or a high humidity environment. ${ }^{25,26}$ The sacrificial template technique used in this study does not require the same level of strict processing conditions (e.g., controlled humidity, specialized equipment), with the pattern of the PDA film amenable to simple adjustments by varying template particle size and/or shape.

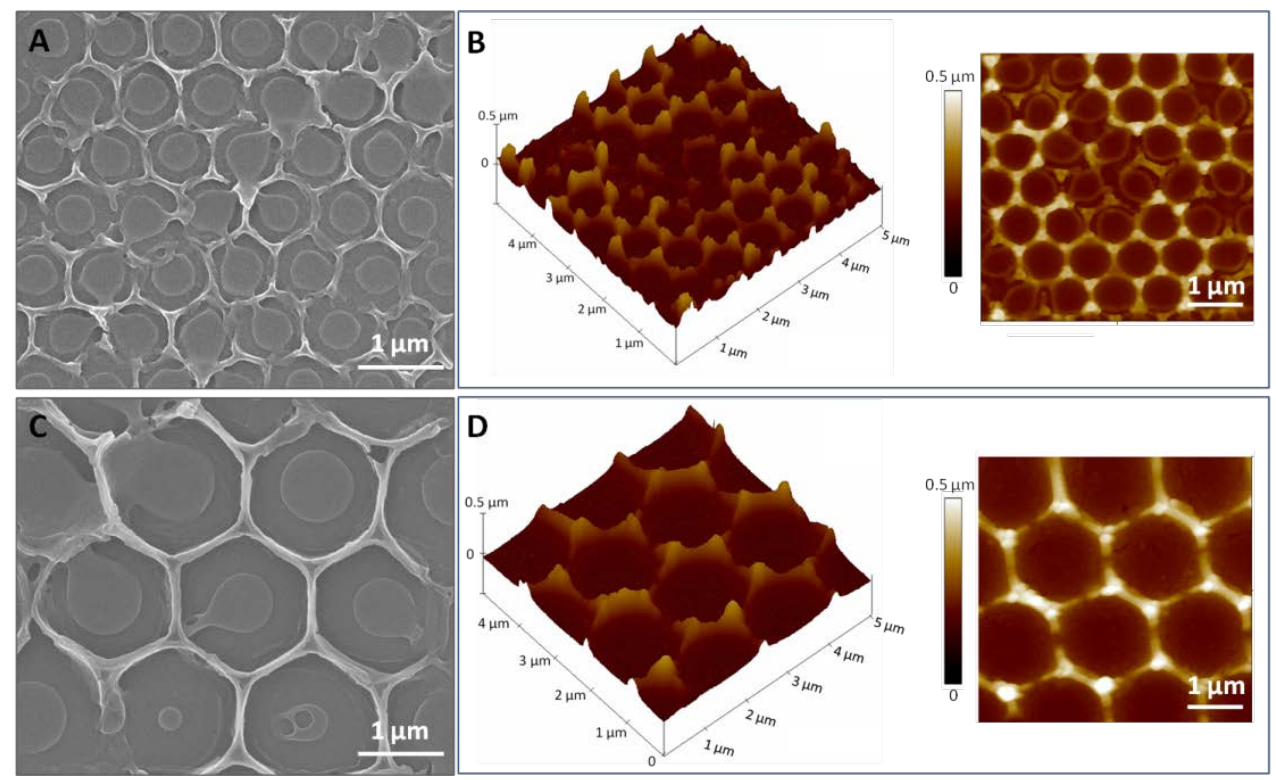

Figure 2. SEM images (A, C) and AFM images (B, D) of patterned PDA films derived from different size PS template particles (A, B derived from PS particles with diameters of $0.8 \mu \mathrm{m}$, and C, D derived from PS particles with diameters of $1.7 \mu \mathrm{m})$. See Figures S1-S3 for additional details. 
The ability of the patterned PDA films to function as cell culture substrates was investigated by incubation with cells from either a human cervical cancer cell line (HeLa) or a rat mesenchymal stem cell line. Cell viability was quantified using a cell viability assay and was found to be around or over $90 \%$ for both cell types and all the film types investigated (Figures 3 and S4). Importantly, no significant differences were observed between the patterned and the non-patterned films or between the different patterned films (Figure 3). Cell morphology was investigated using fluorescence microscopy and no substantial differences were observed when comparing the non-patterned and the patterned films or the different patterned films for each cell type (Figure S5).

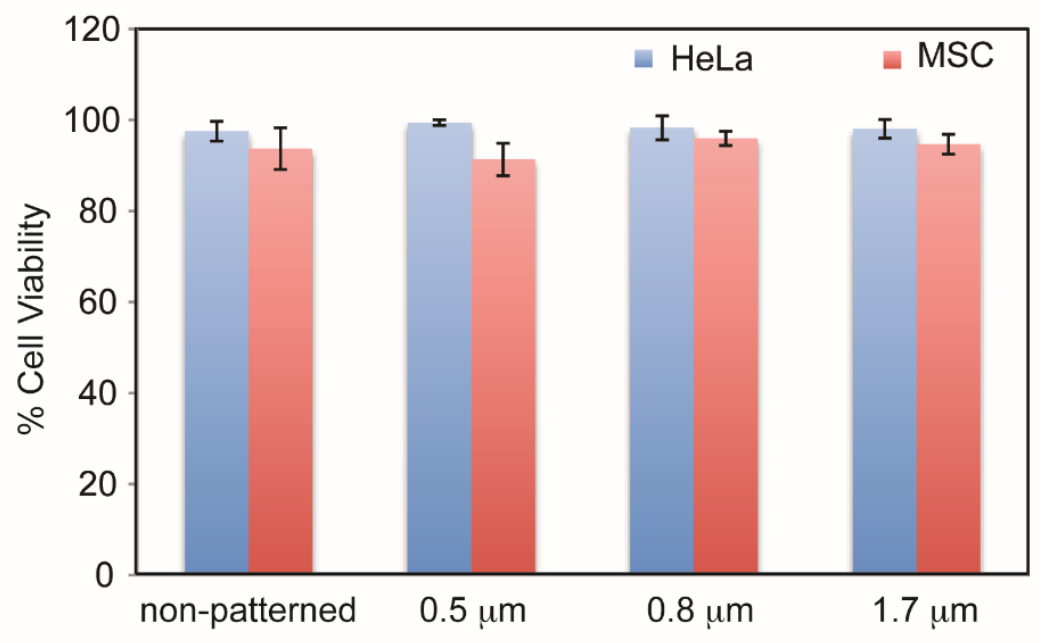

Figure 3. The viability of HeLa cells and mesenchymal stem cells grown on non-patterned and patterned PDA films prepared using $0.5 \mu \mathrm{m}, 0.8 \mu \mathrm{m}$ and $1.7 \mu \mathrm{m}$ PS templates. The experiments were performed in duplicate with the error bars indicating average \pm standard deviation.

Cell adhesion was quantified using fluorescence microscopy. Cell adhesion on nonpatterned PDA films was used as controls. For HeLa cells, a 43\% increase in the number of cells adhered to the $0.5 \mu \mathrm{m}$-patterned films $\left(130 \pm 13\right.$ cells $\left.\mathrm{cm}^{-2}\right)$ compared with the nonpatterned PDA films (91 \pm 12 cells $\mathrm{cm}^{-2}$ ) was observed (Figure 4A, B and C). This demonstrates that cell attachment can be enhanced through the use of grooved PDA films. 
Our results show the same trend as that obtained using "micro-wavy" patterned surfaces, where the patterned surfaces demonstrated increased cell adhesion compared to flat surfaces. ${ }^{27}$ A possible explanation for the increase in cell adhesion could be the increase in film contact area available to the cells for the patterned films, as has been discussed previously (although different film morphologies can cause different cell responses). ${ }^{28} \mathrm{We}$ also observed $\sim 64 \%$ and $~ 24 \%$ increases in HeLa cell adhesion on $0.8 \mu$ m-patterned and 1.7 $\mu$ m-patterned PDA films, respectively, compared with non-patterned PDA films (Figure 5A). These results suggest that the groove size of the PDA film is not a dominant factor for governing HeLa cell adhesion.
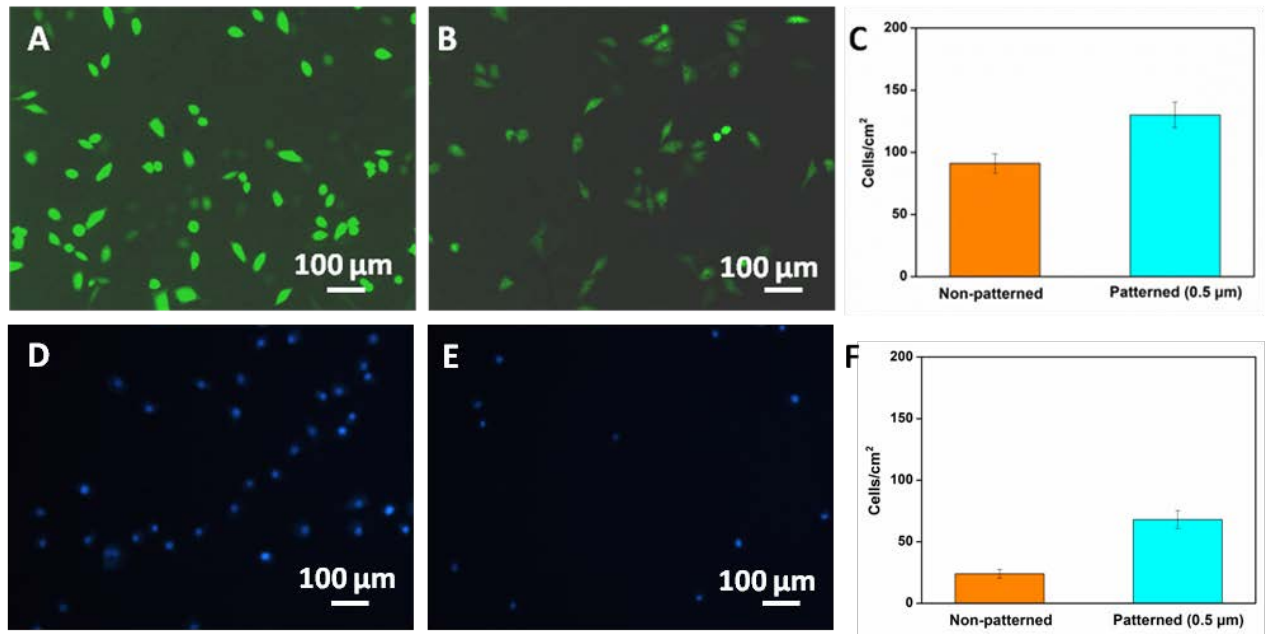

Figure 4. Fluorescence microscopy images of $\operatorname{HeLa-GFP~(A,~B)~and~mesenchymal~stem~cells~(D,~E,~}$ with Hoechst-stained nuclei) cultured on: (A, D) $0.5 \mu \mathrm{m}$-patterned PDA films and (B, E) nonpatterned PDA films. Each image represents an area of $900 \mu \mathrm{m} \times 670 \mu \mathrm{m}$. (C, F) Quantification of attached HeLa-GFP cells (C) and stem cells (F) per $\mathrm{cm}^{2}$ on non-patterned PDA, and on patterned PDA.

We also examined the adhesion of mesenchymal stem cells on the $0.5 \mu \mathrm{m}$-patterned PDA films, and a substantial increase ( 183\%) of the number of stem cells attached (68 \pm 7 cells $\left.\mathrm{cm}^{-2}\right)$ compared with non-patterned film $\left(24 \pm 4\right.$ cells $\left.\mathrm{cm}^{-2}\right)$ was observed (Figure 4D, E and F). Likewise, we observed $\sim 204 \%$ and $~ 313 \%$ increases in the number of mesenchymal 
stem cells adhered to the $0.8 \mu \mathrm{m}$-patterned and $1.7 \mu \mathrm{m}$-patterned films, respectively, compared with non-patterned PDA films (Figure 5B). Stem cells have previously been shown to exhibit a high affinity to PDA-based scaffolds, resulting in a significant enhancement in cell adhesion. ${ }^{29}$ In one study, up to $20 \%$ increase in proliferation was observed from adiposederived stem cells grown on PDA functionalized PLGA compared with PLGA alone. ${ }^{30}$ Our work demonstrates that having a patterned, or specifically, grooved PDA films can further improve cell attachment by up to 300\%, depending on cell type and surface groove size.

A

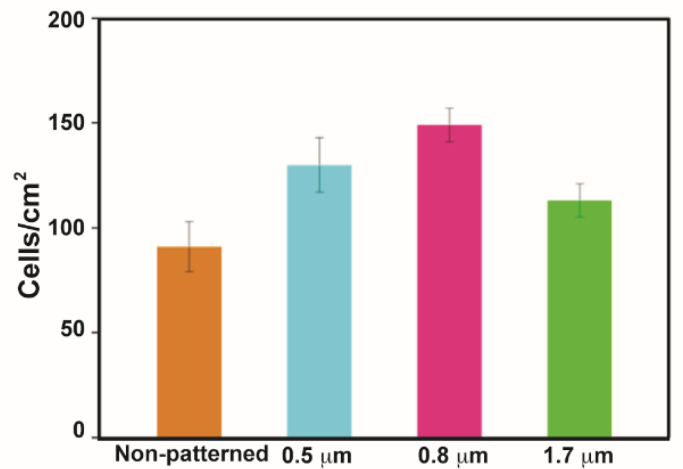

B

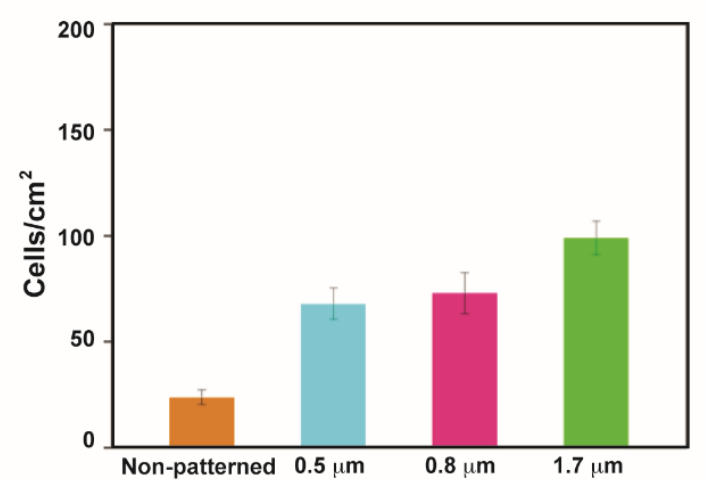

Figure 5. Quantification of adhered (A) HeLa cells, and (B) mesenchymal stem cells per $\mathrm{cm}^{2}$ on nonpatterned and patterned PDA films prepared using $0.5 \mu \mathrm{m}, 0.8 \mu \mathrm{m}$ and $1.7 \mu \mathrm{m}$ PS templates. The experiments were performed in triplicate with the error bars indicating average \pm standard deviation.

In summary, we prepared engineered PDA films with controllable surface patterns using a templated polymerization approach at the air/water interface. The pattern size and the resulting topography of the PDA films could be tuned by simply varying the template size. Cell adhesion studies using cancer cells and mesenchymal stem cells demonstrated that the patterned PDA surface significantly enhanced the adhesion of both cells. While the current technique and protocol was used to prepare films using particle templates ranging from 0.5 to $1.7 \mu \mathrm{m}$ in diameter (with the smoothest films resulting from preparation with the $0.5 \mu \mathrm{m}$ particle templates), we are currently exploring strategies to prepare films using smaller and 
larger templates, as well as films with increased or decreased stability (e.g., using shorter or longer polymerization times). ${ }^{24}$ These results, combined with the biocompatible nature of the material and ease of production of the patterned films, highlight a potential in biomaterial research as well as in various biomedical applications, such as for regenerative medicine and tissue engineering.

\section{Experimental Procedures}

Materials. Polystyrene (PS) particles with diameters of 0.53 and $1.70 \mu \mathrm{m}$ were purchased from Spherotech (US). PS particles with a diameter of $0.84 \mu \mathrm{m}$ were purchased from MicroParticles GmbH (Berlin, Germany). Hydroxytyramine hydrochloride (dopamine) and tetrahydrofuran (THF) were obtained from Sigma-Aldrich (Castle Hill, Australia). Tris(hydroxymethyl)-aminomethane (Tris) and ethanol were obtained from Chem-Supply (Australia). Cell culture medium Dulbecco's Modified Eagle Medium (DMEM), heatinactivated fetal bovine serum (HI-FBS), a LIVE/DEAD viability/cytotoxicity kit (containing the fluorescent dyes calcein-AM and ethidium homodimer-1), and Dulbecco's PhosphateBuffered Saline (DPBS) were purchased from Thermo Fisher Scientific (Scoresby, Australia). A MycoAlert mycoplasma detection kit was purchased from Lonza (Australia). High-purity (Milli-Q) water was prepared by a Millipore Milli-Q water purification system with a resistivity greater than $18 \mathrm{M} \Omega \cdot \mathrm{cm}$. Silicon wafers (MMRC Pty Ltd, Australia) were cut into $1.5 \times 1.5 \mathrm{~cm}$ slides and cleaned with Piranha solution (7:3 v/v mixture of concentrated sulfuric acid and 30\% hydrogen peroxide. Caution! Piranha solution is highly corrosive and extreme care should be taken during preparation and operation.

Synthesis of PDA Films. To set up the experiment, a silicon wafer was fixed in the center of the glass petri dish and Milli-Q water was slowly dispensed dropwise into the petri dish around the silicon wafer until the level of the water was above the surface of the silicon 
wafer but not covering it. A suspension of PS template particles $\left(2 \mathrm{mg} \mathrm{mL}^{-1}\right.$ in a $50 \mathrm{wt} \%$ ethanol solution) was sonicated for $2 \mathrm{~h}$, followed by addition of $35 \mu \mathrm{L}$ of the particle suspension onto the middle of the silicon wafer. The PS particles quickly spread onto the surface of the silicon wafer, forming an array and assembling as a monolayer on the water surface. Milli-Q droplets were added carefully into the petri dish until the silicon wafer plate was fully covered with the liquid solution to allow the PS particles to float from the hydrophilic substrate to the air/water interface. DA was dissolved in Tris buffer (50mM, pH 10.5) and then immediately injected into the bulk solution in the dish (the final working concentration of DA was $0.3 \mathrm{mg} \mathrm{mL}^{-1}$ ). The dish was left to stand for $16 \mathrm{~h}$ to allow the spontaneous polymerization of DA. For the synthesis of non-patterned PDA films, cover glasses were immersed into Tris buffer (50mM, pH 10.5), followed by the addition of DA into the buffer (the final working concentration of DA was $0.3 \mathrm{mg} \mathrm{mL}^{-1}$ ). Polymerization was allowed to proceed for $16 \mathrm{~h}$. The films were transferred from the petri dishes (used during synthesis) to the multiwell plates (used for cell studies) by gently inserting a substrate (e.g., coverslip) next to the prepared films, allowing the substrate to sink to the bottom of the petri dish, and then positioning the substrate directly below the prepared films floating at the air/water interface. (This can most easily be achieved by using petri dishes larger than the films that need to be prepared-e.g., to prepare a $5 \times 5 \mathrm{~cm}$ film, use a $10 \mathrm{~cm}$ in diameter petri dish so that the substrate can be easily inserted without disturbing the film. The substrates/coverslips can also be added at the very beginning into the empty petri dish and left at the bottom of the petri dish during the whole procedure.) The film is then deposited onto the substrate/coverslip by slowly aspirating all of the solution so that the floating film moves downward (as the air/liquid interface moves toward the bottom of the petri dish) until all liquid is removed and the film is deposited on top of the substrate/coverslip. The assembled films have optical properties and the position of the floating films can therefore 
easily be tracked by observing interference colors ("rainbow colors”) using the naked eye when gently tilting the petri dish. ${ }^{24}$

Characterization. The surface topography of patterned PDA films was observed by using field emission scanning electron microscopy (JSM-7600F, JEOL) and atomic force microscopy (AFM, Dimension 3100, Veeco) in tapping mode under dry conditions.

Cell Culture. HeLa cells were cultured in DMEM (with GlutaMAX supplement) containing $10 \%$ of $\mathrm{FBS}$ at $37{ }^{\circ} \mathrm{C}$ in a $5 \% \mathrm{CO}_{2}$ humidified atmosphere and subcultured prior to confluence using trypsin. Rat mesenchymal stem cells (Gibco, Thermo Fisher Scientific) were cultured in DMEM supplemented with 10\% MSC-qualified FBS. Cells were cultured without antibiotics and with no sign of bacterial infection. The cell cultures also tested negative for mycoplasma infection (MycoAlert kit).

Cell Studies. Cells were maintained at $37{ }^{\circ} \mathrm{C}$ with $5 \% \mathrm{CO}_{2}$ in DMEM containing $10 \%$ of FBS. Cover glasses (diameter $18 \mathrm{~mm}$ ) were cleaned with Piranha solution for $20 \mathrm{~min}$, and then rinsed with copious amount of Milli-Q water. Caution! Piranha solution is highly corrosive and extreme care should be taken during preparation and operation. PDA films were prepared, as described above, and then transferred into a 12-well plate for cell adhesion studies. Prior to the addition of cells, glass slides were pre-treated with cell culture medium at $37{ }^{\circ} \mathrm{C}$ for $1 \mathrm{~h}$. Each well was cultured with a density of $5 \times 10^{4}$ cells at $37{ }^{\circ} \mathrm{C}$ in $5 \% \mathrm{CO}_{2}$. After 24 h incubation, un-attached cells were removed by washing with DPBS three times. For mesenchymal stem cells, the cell nuclei were subsequently stained with Hoechst 33342 (10 $\mu \mathrm{g} \mathrm{mL}^{-1}$ ) for $30 \mathrm{~min}$, followed by washing with DPBS (three times). The HeLa-GFP cells used were from a HeLa cell line stably transfected to express green fluorescent protein (GFP) and therefore did not need further fluorescent labeling. The number of attached cells was quantified by acquiring 10 images from random locations on each substrate and counting the 
cells manually. Separately, but following the same procedure to prepare samples of cells cultured on films, cell viability was assessed using a cell viability assay (LIVE/DEAD viability/cytotoxicity kit) following the manufacturer's instructions. Cell viability was quantified by taking 10 microscopy images of live/dead stained cells from random locations using fluorescence microscopy and counting the number of cells stained as "live" (green, calcein-AM) or "dead" (red, ethidium homodimer-1). For the cell viability studies mesenchymal cells and HeLa cells without GFP were used. Cells were imaged using fluorescence microscopy (Olympus IX71) with corresponding fluorescence filter set.

\section{Acknowledgments}

This research was conducted and funded by the Australian Research Council (ARC) Centre of Excellence in Convergent Bio-Nano Science and Technology (project number CE140100036). This work was also supported by the ARC under the Australian Laureate Fellowship (F.C., FL120100030) and Super Science Fellowship (F.C., FS110200025) schemes. This work was performed in part at the Materials Characterisation and Fabrication Platform (MCFP) at the University of Melbourne and the Victorian Node of the Australian National Fabrication Facility (ANFF). We thank Biao Kong (the University of Melbourne) and Wei Zhu (Tsinghua University) for helpful discussions. We thank Peggy Chan (Swinburne University) for the mesenchymal stem cells.

Supporting Information. Additional AFM analysis, additional SEM images, and additional cell viability and morphology microscopy images.

\section{References}

1. Van Vlierberghe, S., Dubruel, P., and Schacht, E. (2011) Biopolymer-based hydrogels as scaffolds for tissue engineering applications: a review. Biomacromolecules 12, 1387-1408. 
2. Bacakova, L., Filova, E., Rypacek, F., Svorcik, V., and Stary, V. (2004) Cell adhesion on artificial materials for tissue engineering. Physiol. Res. 53 (Suppl 1), S35-45.

3. Lutolf, M.P., and Hubbell, J.A. (2005) Synthetic biomaterials as instructive extracellular microenvironments for morphogenesis in tissue engineering. Nat. Biotechnol. 23, 47-55.

4. Stevens, M. M., and George, J. H. (2005) Exploring and engineering the cell surface interface. Science 310, 1135-1138.

5. Bacakova, L., Filova, E., Parizek, M., Rumi, T., and Svorcik, V. (2011) Modulation of cell adhesion, proliferation and differentiation on materials designed for body implants. Biotechnol. Adv. 29, 739-67.

6. Anselme, K., Ploux, L., and Ponche, A. (2010) Cell/material interfaces: influence of surface chemistry and surface topography on cell adhesion. J. Adhes. Sci. Technol. 24, 831-852.

7. Dalby, M. J., Gadegaard, N., and Oreffo, R. O. C. (2014) Harnessing nanotopography and integrin-matrix interactions to influence stem cell fate. Nat. Mater. 13, 558-569.

8. Biggs, M. J. P., Richards, R. G., and Dalby, M. J. (2010) Nanotopographical modification: a regulator of cellular function through focal adhesions. Nanomed. Nanotechnol. Biol. Med. 6, 619-633.

9. Bidanset, D. J. LeBaron, R. Rosenberg, L. Murphy-Ullrich, J. E., and Hook, M. (1992) Regulation of cell substrate adhesion: effects of small galactosaminoglycan-containing proteoglycans. J. Cell Biol. 118, 1523-1531.

10. Best, J. P., Javed, S., Richardson, J. J., Cho, K. L., Kamphuis, M. M. J., and Caruso, F. (2013) Stiffness-mediated adhesion of cervical cancer cells to soft hydrogel films. Soft Matter 9, 4580-4584.

11. Chen, S., Lu, X., Hu, Y., and Lu, Q. (2015) Biomimetic honeycomb-patterned surface as the tunable cell adhesion scaffold. Biomater. Sci. 3, 85-93.

12. Sjöström, T., Dalby, M. J., Hart, A., Tare, R., Oreffo, R. O. C., and Su, B. (2009) Fabrication of pillar-like titania nanostructures on titanium and their interactions with human skeletal stem cells. Acta Biomater. 5, 1433-1441.

13. Curtis, A. S. G., Casey, B., Gallagher, J. O., Pasqui, D., Wood, M.A., and Wilkinson, C. D. W. (2001) Substratum nanotopography and the adhesion of biological cells. Are symmetry or regularity of nanotopography important? Biophys. Chem. 94, 275-283. 
14. Teixeira, A. I., Nealey, P. F., and Murphy, C. J. (2004) Responses of human keratocytes to micro- and nanostructured substrates. J. Biomed. Mater. Res. 71A, 369-376.

15. Lynge, M. E., Schattling, P., and Städler, B. (2015) Recent developments in poly (dopamine)based coatings for biomedical applications. Nanomedicine 10, 2725-2742.

16. Lee, H., Dellatore, S. M., Miller, W. M., and Messersmith, P. B. (2007) Mussel-inspired surface chemistry for multifunctional coatings. Science 318, 426-430.

17. Madhurakkat Perikamana, S. K., Lee, J., Lee, Y. B., Shin, Y. M., Lee, E. J., Mikos, A. G. and Shin, H. (2015) Materials from mussel-inspired chemistry for cell and tissue engineering applications. Biomacromolecules 16, 2541-2555.

18. Postma, A., Yan, Y., Wang, Y., Zelikin, A. N., Tjipto, E., and Caruso, F. (2009) Selfpolymerization of dopamine as a versatile and robust technique to prepare polymer capsules. Chem. Mater. 21, 3042-3044.

19. Cui, J., Yan, Y., Such, G. K., Liang, K., Ochs, C. J., Postma, A., and Caruso, F. (2012) Immobilization and intracellular delivery of an anticancer drug using mussel-inspired polydopamine capsules. Biomacromolecules 13, 2225-2228.

20. Chen, X., Yan, Y., Müllner, M., van Koeverden, M. P., Noi, K. F., Zhu, W., and Caruso, F. (2014) Engineering fluorescent poly(dopamine) capsules. Langmuir 30, 2921-2925.

21. Tsai, W. -B., Chen, W. -T., Chien, H. -W., Kuo, W. -H., and Wang, M. -J. (2011) Poly(dopamine) coating of scaffolds for articular cartilage tissue engineering. Acta Biomater. 7, 41874194.

22. Liu, Y. -T., Kung, K. -C., Yang, C. -Y., Lee, T. -M., and Lui, T. -S. (2014) Engineering threedimensional structures using bio-inspired dopamine and strontium on titanium for biomedical application. J. Mater. Chem. B 2, 7927-7935.

23. Yang, K., Lee, J. S., Kim, J., Lee, Y. B., Shin, H., Um, S. H., Kim, J. B., Park, K. I., Lee, H., and Cho, S.-W. (2012) Polydopamine-mediated surface modification of scaffold materials for human neural stem cell engineering. Biomaterials 33, 6952-6964. 
24. Zhu, W., Yang, H., Lan, Y., Yin, X., Wang, S., Wang, C., Gao, N., and Li, G. (2016) Photonic Janus films with highly tunable Janus balance. Adv. Mater. Interfaces 1600225. doi:10.1002/admi.20060225

25. Escalé, P., Rubatat, L., Billon, L., and Save, M. (2012) Recent advances in honeycombstructured porous polymer films prepared via breath figures. Eur. Polym. J. 48, 1001-1025.

26. Widawski, G. Rawiso, M., and Francois, B. (1994) Self-organized honeycomb morphology of star-polymer polystyrene films. Nature 369, 387-389.

27. Hu, J., Hardy, C., Chen, C. -M., Yang, S., Voloshin, A. S., and Liu, Y. (2014) Enhanced cell adhesion and alignment on micro-wavy patterned surfaces. PLOS One 9, e104502

28. Yao, H., Peng, R., and Ding, J. (2013) Cell-material interactions revealed via material techniques of surface patterning. Adv. Mater. 25, 5257-5286.

29. Ko, E., Yang, K., Shin, J., and Cho, S. -W. (2013) Polydopamine-assisted osteoinductive peptide immobilization of polymer scaffolds for enhanced bone regeneration by human adiposederived stem cells. Biomacromolecules 14, 3202-3213.

30. Yang, K., Lee, J. S., Kim, J., Lee, Y. B., Shin, H., Um, S. H., Kim, J. B., Park, K. I., Lee, H., and Cho, S. -W. (2012) Polydopamine-mediated surface modification of scaffold materials for human neural stem cell engineering. Biomaterials 33, 6952-6964. 


\section{TOC Figure}

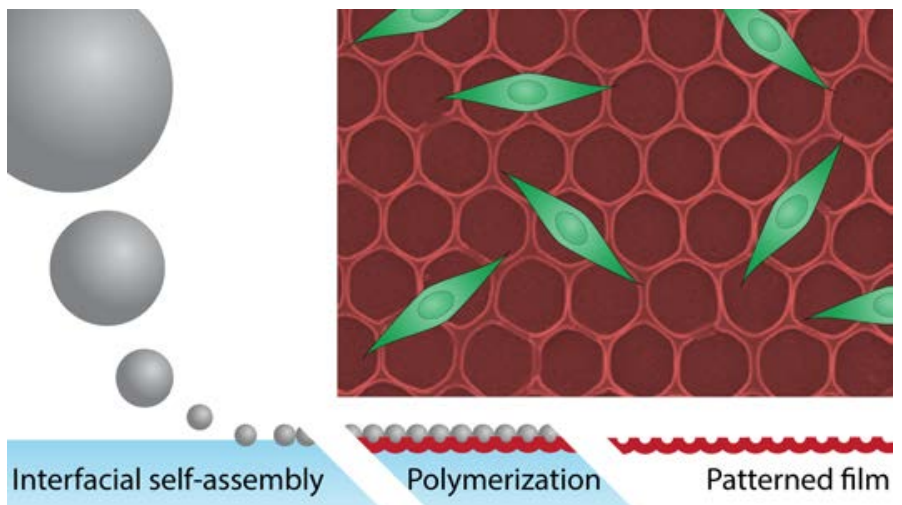




\section{University Library}

\section{- M M N E R VA A gateway to Melbourne's research publications}

Minerva Access is the Institutional Repository of The University of Melbourne

\section{Author/s:}

Chen, X;Cortez-Jugo, C;Choi, GH;Bjornmalm, M;Dai, Y;Yoo, PJ;Caruso, F

Title:

Patterned Poly(dopamine) Films for Enhanced Cell Adhesion

Date:

2017-01-01

Citation:

Chen, X., Cortez-Jugo, C., Choi, G. H., Bjornmalm, M., Dai, Y., Yoo, P. J. \& Caruso, F. (2017). Patterned Poly(dopamine) Films for Enhanced Cell Adhesion. BIOCONJUGATE CHEMISTRY, 28 (1), pp.75-80. https://doi.org/10.1021/acs.bioconjchem.6b00544.

Persistent Link:

http://hdl.handle.net/11343/123197 